HRJ

V.2 n.11 (2021)

Recebido: 30/11/2020

Aceito: 22/04/2021

\title{
Assistência de Enfermagem aos pacientes de Cuidados Paliativos Oncológicos
}

\author{
Pâmela Rodrigues da Silva ${ }^{1}$ \\ Felipe Amorim Tavares Favilla ${ }^{2}$ \\ Alexandra I. Amorim Lino ${ }^{3}$
}

${ }^{1}$ ESCS -Escola Superior de Ciências da Saúde pamelards.bem@gmail.com

${ }^{2}$ Hospital de Apoio - DF tavaresfavilla@gmail.com

3Instituto de Gestão Estratégica de Saúde do Distrito Federal - IGESDF alexandra.lino2@ gmail.com

\section{RESUMO}

Este estudo teve como objetivo compreender como se desenvolve a assistência de enfermagem prestada aos pacientes em cuidados paliativos e avaliar a utilização dos escores prognósticos Palliative Prognostic Index, a partir de uma revisão integrativa. Encontrou-se um total de 180 referências bibliográficas, após análise, restando 45 publicações. Notou-se que algumas dificuldades a uma assistência de qualidade aos pacientes vêm desde o processo de formação da enfermagem e evidenciando que o escore é versátil e de fácil aplicação.

Palavras-chave: Cuidados de enfermagem; Cuidados paliativos; Oncologia; Enfermagem de Cuidados paliativos na terminalidade da vida; Índice de Prognóstico Paliativo.

\section{Nursing Assistance to Oncology Palliative Care patients}

\section{ABSTRACT}

This study aimed to understand how nursing care provided to patients in palliative care is develops and evaluate the use of prognostics scores Palliative Prognostic Index, from an integrative review. Found himself of 180 bibliographic references were found, after analysis remaing 45 publications. It was noted that some difficulties in providing quality care to pacients come from the nursing training process, showing the score is versatile and easy to apply.

Keywords: Nursing care; Palliative care; Oncology; Palliative Care Nursing at the end of life; Palliative prognostic Index.

\section{INTRODUÇÃO}

Nas últimas décadas, o Brasil tem sofrido importantes transformações no seu padrão demográfico e no perfil de morbidade e mortalidade da população. A redução da fertilidade, a urbanização crescente, o aumento da esperança de vida ao nascer e o envelhecimento 
populacional modificaram o perfil epidemiológico do país, causando declínio das doenças infecciosas, aumento das causas externas e predomínio das doenças crônicas não transmissíveis, representando um dos grandes desafios a serem enfrentados, tanto no âmbito científico, como nas políticas públicas ${ }^{1}{ }^{2}, 3$.

Os avanços científicos e tecnológicos ocorridos nas últimas décadas do século $\mathrm{XX}$, associados ao desenvolvimento da terapêutica, fizeram com que muitas doenças mortais se transformassem em doenças crônicas, contribuindo, assim, para a longevidade ${ }^{4}, 5$.

As doenças crônicas, tais como doenças do aparelho circulatório, câncer, doenças respiratórias crônicas e diabetes, perduram por longos períodos de tempo, com múltiplos problemas coexistentes e dependência progressiva, sendo necessária a promoção de cuidados intensos ${ }^{1,3}$.

Com este novo perfil epidemiológico, percebe-se que, mesmo não havendo cura para determinada patologia, existe a possibilidade de atendimento direcionado a manter ou melhorar a qualidade de vida através dos cuidados aos pacientes e familiares ${ }^{2}, 5$.

Nos Cuidados Paliativos são prioridades: as ações em saúde, o alívio da dor, a redução do sofrimento e a oferta de cuidado para aqueles que não podem ser curados. Trata-se de uma abordagem de cuidado diferenciada que visa a qualidade de vida a pacientes e suas famílias, que enfrentam doenças que ameaçam a vida, através da prevenção e alívio do sofrimento, provendo identificação precoce, avaliação e tratamento da dor e outros distúrbios de natureza física, psicossocial e espiritual ${ }^{6,7,8}$.

A habilidade de elaborar prognósticos é uma tarefa pouco entendida e pouco valorizada. Muitos pacientes e familiares desejam informações sobre expectativa de vida e os profissionais de saúde envolvidos no cuidado paliativo têm a incumbência de estar preparados para responder a essa demanda. Dentre as tarefas das equipes de cuidado paliativo, está a 
discussão sobre prognósticos e objetivos da assistência, o que tem sido sistematicamente negligenciado por profissionais de saúde de outras especialidades ${ }^{6},{ }^{9},{ }^{10}$.

Uma ferramenta clínica que tem grande potencial para ser utilizada no sentido de estabelecer prognóstico desses pacientes é o Palliative Prognostic Index (PPI) - Índice Prognóstico Paliativo, que se baseia nas seguintes variáveis: Palliative Performance Scale (PPS) - Escala de Desempenho Paliativo, ingesta oral, edema, dispneia em repouso e delirium, a cada qual são conferidos valores. O somatório final do valor de cada variável permite estimar o tempo restante de vida ${ }^{11}, 12$.

Assim, o cuidado paliativo deve ser iniciado o mais precocemente possível, juntamente com outras medidas de prolongamento da vida, como a quimioterapia e a radioterapia e incluir todas as investigações necessárias para melhor compreender e controlar situações clínicas estressantes ${ }^{6},{ }^{13}$.

A enfermagem tem papel fundamental nos cuidados paliativos visto que o enfermeiro tem como objeto de trabalho o cuidado, estabelecendo/mantendo o vínculo e facilitando a promoção da saúde e do bem estar biopsicossocial, conduzindo pacientes e familiares às melhores formas de enfrentamento do processo de doença e morte ${ }^{14}, 15,16$.

Inserido na equipe multidisciplinar, é papel do enfermeiro atuar em busca da comunicação eficaz, aberta e adaptada ao contexto terapêutico, visando à negociação de metas assistenciais acordadas com o paciente e sua família de modo a coordenar o cuidado planejado ${ }^{14}, 16$.

O enfermeiro deve conhecer e dominar o manejo da dor e outros sintomas, saber identificar as necessidades sociais, espirituais e psicológicas do paciente e estabelecer uma relação terapêutica efetiva e, para tanto, necessita da comunicação ${ }^{17},{ }^{18}$. 
Diante da responsabilidade frente ao cuidado dos pacientes, a enfermagem se desgasta emocionalmente devido à constante interação com os pacientes enfermos, acompanhando o sofrimento, como a dor, a doença e a morte do ser cuidado ${ }^{8}, 19$.

Então a dificuldade em lidar com a terminalidade da vida e encarar a morte como um processo natural, faz com que parcela dos profissionais de enfermagem encontre alternativas para fugir dessas situações. Assim, alguns evitam os pacientes terminais, não falam com o paciente sobre o processo de doença e morte, não criam vínculos e realizam um cuidado pouco individualizado ${ }^{7}$

Esses fatores comprometem a qualidade da assistência prestada e da comunicação com os pacientes e familiares, sendo objetos de diversos estudos.

\section{MÉTODOS}

\section{Desenho do estudo}

Trata-se de uma revisão com metodologia de um scoping review (análise de escopo). A técnica de scoping review tem a finalidade de sintetizar e de disseminar os resultados de estudos a respeito de um assunto, fornece uma visão descritiva dos estudos revisados, sem avaliá-los criticamente ${ }^{20,21}$.

O levantamento bibliográfico incluiu livros, manuais e artigos sobre Assistência de Enfermagem em Pacientes de Cuidados Paliativos Oncológicos e a utilização do escores prognósticos Palliative Prognostic Index - PPI.

\section{Processo de Revisão}

A busca pelos artigos foi realizada nas bases de dados Scielo, Lilacs e PubMed. após um processo de 4 etapas de filtragem de buscas, foram considerados livros, trabalhos acadêmicos, legislações e artigos, relacionados ao tema, abordado nesta pesquisa. Sendo encontradas 180 referências bibliográficas a partir de 1999, dos quais 45 foram analisadas por estarem relacionadas diretamente ao tema, enfocando selecionadas apenas as que se tratavam 
de estudos relacionados a Cuidados de enfermagem; Cuidados paliativos; Oncologia; Enfermagem de Cuidados paliativos na terminalidade da vida; Índice de Prognóstico Paliativo.

No final das 4 etapas foram excluídos no total de 135 referências, por não relatarem diretamente os cuidados prestados pela enfermagem aos pacientes oncológicos e a utilização do escore PPI no paciente em fim de vida. Procedendo a análise e síntese integrativa da publicação completa das 45 referências restantes.

\section{RESULTADOS E DISCUSSÃO}

Tabela 1 - Resultados dos números de artigos encontrados em cada base de dados, por etapa de pesquisa, maio a agosto de 2020 .

\begin{tabular}{|ccccc|}
\hline Bases de dados & $\mathbf{1}^{\mathbf{a}}$ Etapa $^{\mathbf{a}}$ & $\mathbf{2}^{\mathbf{a}} \mathbf{E t a p a}^{\mathbf{b}}$ & $\mathbf{3}^{\mathbf{a}} \mathbf{E t a p a}^{\mathbf{c}}$ & $\mathbf{4}^{\mathbf{a}}$ Etapa $^{\mathbf{d}}$ \\
\hline Scielo & 90 & 81 & 62 & 27 \\
Lilacs & 47 & 40 & 21 & 10 \\
MEDLINE/ PubMed & 43 & 32 & 15 & 08 \\
\hline Total & 180 & 152 & 98 & 45 \\
\hline
\end{tabular}

O envelhecimento populacional é um fenômeno mundial que, no Brasil e em outros países da América Latina, acontece de forma acelerada. A compressão do processo de envelhecimento faz com que as populações sejam acometidas por uma dupla carga de adoecimento: padecem tanto de doenças agudas, quanto crônicas ${ }^{2}, 22$.

No Brasil, o câncer tem importância epidemiológica, pois se estima para 2020 a predominância do câncer de próstata para casos novos do sexo masculino de 65.840 e para o sexo feminino com o câncer de mama com 66.280 casos novos ${ }^{23}$.

Sendo o câncer uma doença que se caracteriza por um processo de crescimento lógico e coordenado, no qual uma célula normal sofre modificações cumulativas e adquire capacidades especiais até se tornarem malignas ${ }^{13}$. Com um tumor em progressão, com 
instabilidade genética, que possui capacidade de infiltração nos tecidos normais até atingir estruturas adjacentes, disseminando-se metastaticamente aos órgãos e tecidos mais distantes ${ }^{3}$.

A doença oncológica influencia todos os aspectos da vida das pessoas, para além do fisiológico, com comprometimento da individualidade e da sua dignidade, que associado às questões culturais, dimensiona as reações e problemas concretos como a dor, limitações físicas e emocionais, o desfiguramento e a finitude, no decorrer do processo de adoecimento $^{22,23}$.

Logo, a assistência ao paciente oncológico envolve conhecimentos técnico científicos, a capacidade e a habilidade de compreender os sentimentos, valores e crenças das pessoas. Assim, a enfermagem encontra-se favorecida diante das possibilidades de intervenções ao paciente oncológico, pois pode aliar conhecimento científico ao contexto das necessidades psicossociais, com resoluções eficientes e humanizadas ${ }^{4},{ }^{8}, 24$.

A atenção dos profissionais de saúde em oncologia foi se expandindo, fazendo com que a prática acadêmica começasse a se tornar atuante na comunidade, favorecendo assim, o crescimento, o desenvolvimento e a ampliação da enfermagem em oncologia, o que estimulou o desenvolvimento desta especialidade. Em 1975, foi criado a Oncology Nursing Society (ONS) nos Estados Unidos, que desde então, lidera as organizações científicas mundiais de enfermagem em oncologia $\mathrm{a}^{25,26}$.

No Brasil, o desenvolvimento acadêmico em enfermagem, na modalidade de pósgraduação latu sensu em oncologia, historicamente teve início com a formação dos Programas de Residência em Enfermagem. O primeiro Programa de Residência desenvolvido no nosso país foi em São Paulo, no Hospital Infantil do Morumbi, em 1961'26,27.

Hoje, a valorização dos profissionais de saúde especializados em oncologia implica em trabalhar com a qualidade de vida, focalizando as dimensões físicas, psicológicas e sociais dos pacientes ${ }^{16}$. 


\section{Cuidados Paliativos e Assistência de Enfermagem}

Em face de doenças ameaçadoras da vida, indica-se a implementação de Cuidados Paliativos desde o momento do diagnóstico, concomitante ao tratamento curativo apropriado ${ }^{6}$.

O conceito de cuidados paliativos evoluiu ao longo do tempo à medida que esta filosofia de cuidado foi se desenvolvendo em muitas regiões do mundo. Sua origem se deu na Inglaterra, por Cicely Saunders, fundadora do St. Christopher Hospice em Londres, cujo objetivo era o de afirmar o cuidado daqueles que se aproximavam da morte ${ }^{3}, 6,28$.

Preocupada com o descaso social em que viviam os pacientes em fase terminal e sua família, Saunders criou e disseminou a filosofia dos cuidados paliativos ${ }^{3},{ }^{6}$. A definição mais recente foi, na qual, trata o cuidado paliativo como uma abordagem assistencial que aumenta a qualidade de vida de pacientes e suas famílias que enfrentam problemas associados com doenças que ameaçam a vida, através de prevenção e alívio do sofrimento ${ }^{15}$.

Na relação entre as pessoas que cuidam e as que são cuidadas, sendo as intervenções técnicas secundárias à relação que se estabelece entre equipe de cuidados e pacientes, a humanização entre a equipe de saúde e o paciente sem possibilidade de cura e a sua família são chave para esse cuidado ${ }^{7}, 29$.

Como princípios dos cuidados paliativos estão: a afirmação da vida e o enfrentamento da morte como um processo natural; o não adiamento e prolongamento da morte; a promoção de alívio da dor e de outros sintomas. Tudo isso integrando oferece um suporte para que os pacientes possam viver o mais ativamente possível nos dias que lhes restam e ajudando a família e os cuidadores no seu processo de 1 luto ${ }^{6},{ }^{30}$.

A assistência paliativa é voltada ao controle de sintomas, sem função curativa, com vistas a preservar a qualidade até o final da vida, possibilitando uma abordagem holística do paciente com doença incurável ${ }^{3},{ }^{18}$.

Representa um conjunto de cuidados prestados ao paciente, desde o início de sua terapêutica, com uma abordagem especializada para ajudar a pessoa a viver melhor e 
favorecer todo e qualquer tratamento que promova qualidade de vida até o momento de sua morte $^{6}, 24,31$.

Estes cuidados pressupõem a ação de uma equipe multiprofissional, já que a proposta consiste em cuidar do indivíduo em todos os aspectos: físico, mental, espiritual e $\operatorname{social}^{3,6,32}$.

A importância da equipe multidisciplinar se dá pelo fato de que os saberes são inacabados, limitados, sempre precisando ser complementados ${ }^{8}$.

O enfermeiro, como membro da equipe multidisciplinar, desempenha um papel fundamental na promoção de cuidados paliativos para os pacientes crônicos - o de minimizar o sofrimento e favorecer qualidade de vida, respaldados na filosofia desses cuidados e em princípios éticos ${ }^{1}, 8$.

Esses princípios fundamentam a prática dos cuidados paliativos e valorizam a autonomia do paciente como um dos pontos fundamentais na busca da excelência dos cuidados prestados pela enfermagem ${ }^{13}, 19$.

$\mathrm{O}$ enfermeiro, enquanto gerente e condutor da equipe de enfermagem e membro de uma equipe multidisciplinar, precisa encarar a finitude e a morte como parte do ciclo vital e, para tanto, necessita de constantes reflexões sobre a terminalidade da vida ${ }^{19}$. Porém, na prática, os profissionais de enfermagem encontram barreiras na assistência ao paciente terminal e seus familiares ${ }^{10}$. Segundo estudos, os enfermeiros relataram dificuldade em entender a morte como um processo natural, motivo que desencadeia uma sensação de frustração, tristeza, impotência e até mesmo culpa por falhas na assistência prestada diante da terminalidade da vida. Nestes casos, a morte é vista como fracasso, pois o que sempre se busca é a melhora do paciente em direção à saúde e nunca em direção contrária ${ }^{15}, 31$.

Se o profissional não consegue alcançar seu objetivo, ou, mais especificamente, se o paciente morre, a atuação pode ser vista por ele e pelos outros como fracassada. Ainda assim, muitos profissionais fazem o que está ao seu alcance para que o paciente tenha qualidade nos 
últimos dias de vida, seja ouvindo os lamentos, histórias ou realizando seus últimos desejos, tornando, de certa forma, o atendimento mais humanizado ${ }^{7}, 9,33$.

A enfermagem é uma das profissões em que ocorre maior desgaste emocional do trabalhador devido à constante interação com seres enfermos, muitas vezes acompanhando o sofrimento, como a dor, a doença e a morte do ser cuidado ${ }^{34}$. O tratamento de pacientes sem possibilidades terapêuticas leva o profissional a confrontar sua finitude com suas limitações e falta de onipotência. Isso gera um misto de sentimentos que variam da culpa, desprezo, tristeza à ansiedade, podendo resultar em um atendimento frio e impessoal, fuga às perguntas do paciente e aos seus pedidos de socorro ${ }^{19},{ }^{34}$.

As dificuldades relatadas pelos enfermeiros em prestar uma assistência de qualidade a pacientes sem possibilidades de cura vem desde o seu processo de formação ${ }^{8}$. Os estudos demonstraram que não existem nos cursos de graduação e pós-graduação programas de estudo voltados para tanatologia e cuidados paliativos, havendo apenas debates superficiais sobre a temática. Isso explicaria o fato dos profissionais transformarem a morte em um tabu e não a aceitarem como um processo natural do ciclo da vida. Muitos mencionam a mesma como um fracasso, fazendo com que encontrem alternativas para não se deparar com a situação: evitam cuidar de pacientes terminais, realizam tratamento pouco individualizado, não criam vínculos com pacientes e familiares, além de fugiram da discussão sobre a morte fazendo promessas de recuperação ao paciente quando se sabe que a morte é praticamente inevitável ${ }^{35},{ }^{36}$.

Outro fator relatado pelos profissionais de enfermagem nos estudos em questão como dificultador no cuidado ao paciente terminal é a ausência da sistematização da assistência. A sistematização da assistência de enfermagem compreende a forma como o trabalho da enfermagem é organizado, de acordo com o método científico e o referencial teórico, de modo que seja possível o melhor atendimento das necessidades de cuidado do indivíduo, família e comunidade. A não sistematização gera uma assistência com ações fragmentadas e 
imediatistas, sem planejamento prévio, comprometendo sua qualidade por não alcançar a integralidade, um ideal do cuidado paliativo, visto que, este valoriza tanto a prevenção e alívio dos sintomas como o apoio às necessidades psicossociais, emocionais e espirituais dos pacientes e familiares $8,10,29,37$.

Com isso, a prática de enfermagem sistematizada se torna fundamental na assistência paliativa, pois favorece a identificação das necessidades de cuidado manifestadas e/ou referidas pelos pacientes e familiares em sua totalidade, bem como a articulação e negociação com os demais membros da equipe de saúde em nome da concretização e melhorias do cuidado, constituindo uma estratégia adequada a uma prática centrada na pessoa e não apenas nas tarefas $8,18,38$.

O emprego adequado da comunicação constitui-se em um dos pilares dos cuidados paliativos e uma medida terapêutica comprovadamente eficaz. Na assistência de enfermagem, a comunicação é vista como uma ferramenta que promove o elo entre a equipe de enfermagem, o paciente e a família, mediante o uso tanto da comunicação verbal quanto da não verbal ${ }^{14,}{ }^{17}$.

Os profissionais de enfermagem devem utilizar a comunicação como instrumento para humanizar o cuidado, uma estratégia de aproximar paciente e equipe, na reconstrução do relacionamento entre o profissional de enfermagem e o ser humano hospitalizado, repercutindo diretamente na qualidade do serviço prestado pelas instituições de saúde e no modo como este é percebido pelo usuário. Apesar de reconhecerem a importância desta técnica, alguns profissionais de enfermagem demonstram não estar preparados para atuar em situações que envolvam a comunicação na terminalidade humana ${ }^{15},{ }^{39}$.

Sabe-se que, o profissional, ao se comunicar com paciente, proporciona um cuidado individual, percebendo o ser humano como ser biológico, psicológico, social e espiritual e não 
como um ser fragmentado em seus sistemas funcionais. Isso possibilita um cuidado integral, obedecendo aos princípios da prática paliativa ${ }^{9},{ }^{14}$.

Após análise da assistência da enfermagem prestada ao paciente oncológico, notou-se importantes aspectos, além do estudo da pesquisa.

\section{Palliative Prognostic Index (PPI)}

No contexto dos Cuidados Paliativos, um prognóstico acurado é precioso para implementar terapêuticas e orientar na tomada de decisões, aplicando os esforços mais adequados a cada situação. A demanda por expectativas de sobrevida em pacientes de prognóstico reservado alavancou pesquisas na área, resultando na publicação de diversos Escores Preditores de Mortalidade ${ }^{12}$.

Escores Preditores de Mortalidade são ferramentas que ajudam o profissional de saúde a estimar a sobrevida de um paciente, através da análise e pontuação de parâmetros clínicos e/ou laboratoriais. A realização de prognósticos assertivos é um desafio para o corpo clínico, seja pela ausência de disciplinas que abordem esse conteúdo durante a formação acadêmica, seja pela disponibilidade de instrumentos validados e de qualidade. Visando sanar esse problema, a Associação Européia de Cuidados Paliativos (EAPC), recomenda a utilização do Palliative Prognostic Score (PaP) ou do Palliative Prognostic Index (PPI) aliada a expectativa de vida estimada pelo clínico de referência ${ }^{40}$.

O escore Palliative Prognostic Index (PPI) foi derivado e validado com sucesso em um estudo de coorte, que avaliou pacientes com doença maligna avançada, internados em um hospital no Japão. O mesmo estudo foi submetido a nova validação na Irlanda, novamente com êxito, que incluiu 195 pacientes internados ou ambulatoriais ${ }^{11}$.

O PPI é um escore que atribui diferentes pesos para os seguintes itens: Palliative Performance Scale (PPS), aceitação da dieta oral, presença de edema, dispneia em repouso e delirium. O PPI diferencia-se dos demais escores preditores pois não se utiliza de exames laboratoriais ou estimativas do médico assistente para seu cálculo. Os achados pontuados são 
exclusivamente clínicos, tornando a aplicação do PPI prático e acessível mesmo em um cenário de escassez de recursos. Pela natureza dos quesitos avaliados no PPI, qualquer integrante da equipe multidisciplinar pode utilizá-lo ${ }^{11},{ }^{12}$.

O surgimento desses sintomas é comum, principalmente nos últimos dias e horas de vida, causando grande sofrimento ao paciente e seus familiares. Os Cuidados Paliativos pautam-se pelo controle eficaz de sintomas, contemplando paciente e familiar em suas diferentes esferas ${ }^{6},{ }^{41}$.

A habilidade de estimar a sobrevida de pacientes oncológicos em cuidados paliativos é relevante, pois, é um fator significativo no momento das tomadas de decisões e na elaboração do plano de cuidado ${ }^{42}$.

O paciente em estado terminal deve ser assistido integralmente, e isto requer complementação de saberes, partilha de responsabilidades, onde demandas diferenciadas se resolvem em conjunto ${ }^{8}, 40$.

O planejamento dessa assistência, baseado em modelos teóricos que se ajustem ao perfil dos pacientes sem possibilidades de cura, é capaz de auxiliar o enfermeiro no estabelecimento das prioridades e no atendimento das necessidades do paciente, possibilitando um cuidado de qualidade ${ }^{43}, 44$.

O paciente acometido por uma doença aguda possui a noção de que a cura parece como meta plausível. No caso do câncer, essa ainda é expressão de horror e catástrofe para o doente e o público leigo. Esta é uma doença crônica de alto custo do tratamento, que implica longevidade dos procedimentos terapêuticos, a perspectiva incerta de cura, queda abrupta na qualidade de vida e perda da autonomia física e psicológica, na maioria das vezes. $\mathrm{Na}$ utilização do escore PPI, a enfermagem consegui melhorar a qualidade de vida do paciente em fim de vida ${ }^{42},{ }^{45}$. 
Para a enfermagem, o paciente oncológico apresenta-se como aquele que demanda cuidados de maior complexidade ${ }^{31}$.

O PPI é um instrumento fácil e de confiável aplicação que não acarreta efeitos colaterais ou ônus financeiro em sua aplicação ${ }^{11},{ }^{12}$. Apesar dos estudos demonstrarem que o PPI se destaca por ser simples, de fácil execução, notou-se que a enfermagem não utiliza de tal ferramenta, para melhoria na qualidade de vida do paciente.

Figura 1. Palliative Prognostic Index - PPI

\begin{tabular}{|c|c|c|c|}
\hline Fator & Escore & Escore total & Sobrevida \\
\hline PPS $10-20 \%$ & 4,0 & Escore $<4,0$ & $>6$ semanas \\
\hline PPS 30-50 & 2,5 & Escore > 4,0 & $<6$ semanas \\
\hline PPS $>50 \%$ & 0 & Escore > 6,0 & $<3$ semanas \\
\hline Delirium & 4,0 & & \\
\hline Dispneia em repouso & 3,5 & & \\
\hline Ingesta oral muito reduzida & 2,5 & & \\
\hline Ingesta oral reduzida & 1,0 & & \\
\hline Ingesta normal & 0 & & \\
\hline Edema & 1,0 & & \\
\hline
\end{tabular}

Quando estipulado como corte o escore 6, o PPI prevê a mortalidade em três semanas, com sensibilidade de $80 \%$ e especificidade de $85 \%$, validado por outros estudos com bons resultados ${ }^{11}$.

\section{Descrição dos Sintomas da Escala PPI}

- PPS 


\begin{tabular}{|c|c|c|c|c|c|}
\hline$\%$ & Deambulação & $\begin{array}{c}\text { Atividade e evidência } \\
\text { de doença }\end{array}$ & Autocuidado & Ingesta & $\begin{array}{c}\text { Nivel da } \\
\text { consciencia }\end{array}$ \\
\hline 100 & Completa & $\begin{array}{l}\text { Atividade normal } \\
\text { e trabalho, sem } \\
\text { evidência de doenca }\end{array}$ & Completo & Normal & Completo \\
\hline 90 & Completa & $\begin{array}{l}\text { Atividade normal } \\
\text { e trabalho, alguma } \\
\text { evidencia de doenca }\end{array}$ & Completo & Normal & Completo \\
\hline 80 & Completa & $\begin{array}{l}\text { Atividade normal } \\
\text { com esforço, alguma } \\
\text { evidencia de doença }\end{array}$ & Completo & $\begin{array}{l}\text { Normal ou } \\
\text { reduzida }\end{array}$ & Completo \\
\hline 70 & Reduzida & $\begin{array}{c}\text { Incapaz para o } \\
\text { trabalho, doença } \\
\text { significativa }\end{array}$ & Completo & $\begin{array}{l}\text { Normal ou } \\
\text { reduzida }\end{array}$ & Completo \\
\hline 60 & Reduzida & $\begin{array}{l}\text { Incapaz para hobbies/ } \\
\text { trabalho doméstico, } \\
\text { doenca significativa }\end{array}$ & $\begin{array}{l}\text { Assistência } \\
\text { ocasional }\end{array}$ & $\begin{array}{l}\text { Normal ou } \\
\text { reduzida }\end{array}$ & $\begin{array}{l}\text { Completo ou } \\
\text { periodos de } \\
\text { confusäo }\end{array}$ \\
\hline 50 & $\begin{array}{l}\text { Maior parte } \\
\text { do tempo } \\
\text { sentado ou } \\
\text { deitado }\end{array}$ & $\begin{array}{c}\text { Incapacitado para } \\
\text { qualquer trabalho, } \\
\text { doença extensa }\end{array}$ & $\begin{array}{l}\text { Assistência } \\
\text { considerável }\end{array}$ & $\begin{array}{l}\text { Normal ou } \\
\text { reduzida }\end{array}$ & $\begin{array}{l}\text { Completo ou } \\
\text { periodos de } \\
\text { confusão }\end{array}$ \\
\hline 40 & $\begin{array}{l}\text { Maior parte } \\
\text { do tempo } \\
\text { acamado }\end{array}$ & $\begin{array}{c}\text { Incapaz para } \\
\text { a maioria das } \\
\text { atividades, doença } \\
\text { extensa }\end{array}$ & $\begin{array}{l}\text { Assistência } \\
\text { quase } \\
\text { completa }\end{array}$ & $\begin{array}{l}\text { Normal ou } \\
\text { reduzida }\end{array}$ & $\begin{array}{l}\text { Completo ou } \\
\text { sonolência, } \\
+/- \text { confusão }\end{array}$ \\
\hline 30 & $\begin{array}{l}\text { Totalmente } \\
\text { acamado }\end{array}$ & $\begin{array}{c}\text { Incapaz para } \\
\text { qualquer atividade. } \\
\text { doenca extensa }\end{array}$ & $\begin{array}{l}\text { Dependencia } \\
\text { completa }\end{array}$ & $\begin{array}{l}\text { Normal ou } \\
\text { reduzida }\end{array}$ & $\begin{array}{l}\text { Completo ou } \\
\text { sonolência, } \\
+/- \text { confusão }\end{array}$ \\
\hline 20 & $\begin{array}{l}\text { Totalmente } \\
\text { acamado }\end{array}$ & $\begin{array}{c}\text { Incapaz para } \\
\text { qualquer atividade, } \\
\text { doenca extensa }\end{array}$ & $\begin{array}{l}\text { Dependência } \\
\text { completa }\end{array}$ & $\begin{array}{c}\text { Minima a } \\
\text { pequenos } \\
\text { goles }\end{array}$ & $\begin{array}{l}\text { Completo ou } \\
\text { sonolência. } \\
+/- \text { confusão }\end{array}$ \\
\hline 10 & $\begin{array}{l}\text { Totalmente } \\
\text { acamado }\end{array}$ & $\begin{array}{l}\text { Incapaz para } \\
\text { qualquer atividade, } \\
\text { doenca extensa }\end{array}$ & $\begin{array}{l}\text { Dependencia } \\
\text { completa }\end{array}$ & $\begin{array}{l}\text { Cuidados } \\
\text { com a } \\
\text { boca }\end{array}$ & $\begin{array}{l}\text { Sonolencia } \\
\text { ou coma, +1- } \\
\text { confusão }\end{array}$ \\
\hline 0 & Morte & - & - & - & - \\
\hline
\end{tabular}

Fonte: Victoria Hospice Socie
Ricardo Tavares de Carvalho.

- Dispneia em repouso

A dispneia pode ser definida como uma experiência subjetiva do desconforto respiratório que consiste em sensações distintas que variam na intensidade ${ }^{3},{ }^{45}$.

Essa experiência deriva da interação entre múltiplos fatores fisiológicos, psicológicos, social e ambiental, podendo induzir respostas fisiológicas e comportamentais secundárias. É um sintoma comum em pacientes com câncer, tanto de origem pulmonar como extrapulmonar, sua prevalência aumenta com a progressão da doença e é presente em $70 \%$ dos pacientes nas últimas seis semanas de $\operatorname{vida}^{3},{ }^{41}$.

- Edema

Edema é o inchaço causado pelo acúmulo anormal de líquido no corpo. O edema é mais comum nos pés e pernas, mas também pode ocorrer nas mãos, braços, rosto, abdome e nos pulmões ${ }^{3},{ }^{41}$. 
- Delirium

Delírio é um problema bastante comum em pessoas com câncer avançado, ocorrendo em $15 \%$ a $30 \%$ dos pacientes internados e em até $85 \%$ das pessoas nas últimas semanas de vida. O delírio pode ser estressante para os pacientes e familiares, podendo inclusive interferir com outros sintomas em tratamento, incluindo o tratamento da dor ${ }^{3}, 6,45$.

É importante esclarecer a diferença entre delírio e demência, pois apresentam sinais comuns. Os pacientes com delírio tornam-se agitados e podem ter perda da consciência ao longo do tempo. A demência desenvolve-se de forma gradual e seus efeitos sobre a memória e a consciência são permanentes ${ }^{3}$.

\section{Limitações do estudo}

Mesmo com uma estratégia de busca abrangente, é possível que alguns estudos relevantes tenham sido perdidos, assim como materiais e pesquisas que não foram publicados em periódicos acadêmicos nas bases pesquisadas.

Por se tratar de uma revisão de escopo não incorpora uma avaliação de qualidade dos estudos para sua inclusão. Assim, os estudos incluídos nesta revisão não foram avaliados por seu rigor científico, e ao decidir resumir e relatar as descobertas gerais sem o escrutínio de um processo de avaliação formal, reconhece-se que se pode ter negligenciado alguns aspectos apresentados nesta revisão.

\section{CONCLUSÕES}

Ao longo do estudo, foi observado que mesmo conhecendo a importância dos cuidados paliativos, os profissionais de enfermagem sentiam dificuldade em criar vínculo e se comunicar com os pacientes e seus familiares durante o processo de terminalidade da vida. Isso se deve ao fato de que a morte é vista por estes profissionais como fracasso, como consequência de falhas na assistência prestada. 
Notou-se dificuldades relatadas pelos enfermeiros em prestar uma assistência de qualidade a pacientes sem possibilidades de cura vem desde o seu processo de formação, devido à inexistência de programas de estudo voltados para cuidados paliativos, havendo apenas debates superficiais sobre a temática. A falta de comunicação entre o profissional, o paciente e seus familiares também foi um ponto negativo. Outro fator relatado como dificultador no cuidado ao paciente terminal é a ausência da sistematização da assistência.

Aliado a isso, a sistematização da assistência de enfermagem possibilitaria um cuidado integral e individualizado, baseado nas necessidades do paciente, alcançando, assim, o ideal dos cuidados paliativos. Com este estudo, não se teve por finalidade produzir um novo conhecimento no âmbito da enfermagem, mas, avivar discussões acerca da assistência prestada aos pacientes em cuidados paliativos, buscando melhor qualidade na promoção do cuidar.

O presente trabalho também se respalda pela importância de obter prognósticos mais assertivos no contexto dos Cuidados Paliativos Oncológicos. Além de ser uma demanda corriqueira por parte de pacientes e familiares, trata-se de um dado fundamental para a priorização de terapêuticas e utilização de recursos.

O PPI, conforme descrito no corpo do trabalho, é um escore versátil e de fácil aplicação. Destaca-se por utilizar parâmetros exclusivamente clínicos, podendo ser aplicado em uma visita à beira-leito por qualquer integrante da equipe interdisciplinar.

\section{REFERÊNCIAS BIBLIOGRÁFICAS}

1. Brasil. Ministério da Saúde. Secretaria de Vigilância em Saúde. Departamento de Análise de Situação de Saúde. Plano de ações estratégicas para o enfrentamento das doenças crônicas não transmissíveis (DCNT) no Brasil 2011-2022 / Ministério da Saúde. Secretaria de Vigilância em Saúde. Departamento de Análise de Situação de Saúde. - Brasília: Ministério 
da Saúde, 2011.

2. Miranda GM, Mendes AC, Silva AL. O envelhecimento populacional brasileiro: desafios e consequências sociais atuais e futuras. Rev bras geriatr gerontol. 2016; 19(3): $507-519$.

3. Otto SE. Oncologia, Rio de Janeiro: Ed. Reichmann \& Affonso. 2002.

4. Secoli SR, Padilha KG, Leite RCBO. Avanços tecnológicos em oncologia: reflexões para a prática de enfermagem. Rev. Bras. Cancerologia, Rio de Janeiro, v.51, n.4, p. 331-37. 2005.

5. Veras R. Envelhecimento populacional contemporâneo: demandas, desafios e inovações. Rev Saúde Pública, 43(3):548-554, 2009.

6. ANCP. Academia Nacional de Cuidados Paliativos. Manual de cuidados paliativos. $2^{\mathrm{a}}$ edição. Rio de Janeiro: Solo, 2012.

7. Hermes HR, Lamarca ICA. Cuidados paliativos: uma abordagem a partir das categorias profissionais de saúde. Ciênc. saúde coletiva, Rio de Janeiro, v.18, n.9, Sept. 2013.

8. Silva, MM, Moreira MC. Sistematização da assistência de enfermagem em cuidados paliativos na oncologia: visão dos enfermeiros. Acta paul. enferm., São Paulo, v.24, n.2, 2011.

9. Andrade CG, Costa SFG, Lopes MEL. Cuidados paliativos: a comunicação como estratégia de cuidado para o paciente em fase terminal. Ciênc. saúde coletiva, Rio de Janeiro, v. 18, n. 9, Sept. 2013.

10. Sales CA et al. Cuidado de enfermagem oncológico na ótica do cuidador familiar no contexto hospitalar. Acta paul. enferm., São Paulo, v. 25, n. 5, 2012.

11. Morita T, Tsunoda J, Inoue S, Chihara S. The Palliative Prognostic Index: a scoring system for survival prediction of terminally ill cancer patients. Supportive Care in Cancer 
1999 Apr 4;7(3):128-33.

12. Carol AS, Eoin T, Barbara AD. Prospective Validation of the Palliative Prognostic Index in Patients with Cancer. Journal of pain and symptom management 35[6], 617-622. 1-6-2008.

13. Lopes A, Nakagawa WT. Conceitos Básicos em Oncologia. São Paulo: Ed. Lemar. 2000.

14. Morais GSN, et al. Comunicação como instrumento básico no cuidar humanizado em enfermagem ao paciente hospitalizado. Acta paul. enferm. São Paulo, v. 22, n.3, June 2009.

15. Fernandes MA et al. Percepção dos enfermeiros sobre o significado dos cuidados paliativos em pacientes com câncer terminal. Ciênc. saúde coletiva, Rio de Janeiro, v.18, n.9, Sept. 2013.

16. Coren, Conselho Regional de Enfermagem de São Paulo. Enfermagem Oncológica: o papel do enfermeiro no tratamento do câncer. Revista Coren, São Paulo, n.57, mai/jun. 2005. 17. Araujo MMT, Silva MJP. A comunicação com o paciente em cuidados paliativos: valorizando a alegria e o otimismo. Rev. esc. enferm. USP, São Paulo, v.41, n.4, Dec. 2007. 18. Lopes RAM, Macedo DD, Lopes MHBM. Diagnósticos de enfermagem mais frequentes em uma unidade de internação de oncologia. Rev. Latino-Am. Enfermagem, Ribeirão Preto, v. 5, n. 4, p. 35-41, Oct. 1997.

19. Avancini BS et al. Cuidados paliativos à criança oncológica na situação do viver/morrer: a ótica do cuidar em enfermagem. Esc. Anna Nery Rev. Enferm;13(4):708716, dez. 2009.

20. Tricco AC, Antony J, Soobiah C, et al. Knowledge synthesis methods for generating or refining theory: a scoping review reveals that little guidance is available. J. clin. Epidemiol. 2016; 73:36-42.

21. Arksey H, O'Malley L. Scoping studies: towards a methodological framework. Int. j. 
soc. res. methodol. 2005; 8:19-32.

22. Fitzmaurice C, Allen C, Barber RM, et al. Global, Regional, and National Cancer Incidence, Mortality, Years of Life Lost, Years Lived With Disability, and DisabilityAdjusted Life-years for 32 Cancer Groups, 1990 to 2015: A Systematic Analysis for the Global Burden of Disease Study. JAMA Oncol. 2019 Apr 1; 3(4): 524-548.

23. Ministério da Saúde. Instituto Nacional do Câncer. Estimativa 2008: Incidência de Câncer no Brasil. Rio de Janeiro: INCA, 2008. 94p.

24. Lopes MHBM et al. Diagnósticos de enfermagem no pósoperatório de mastectomia. Esc. Anna Nery, Rio de Janeiro, v. 17, n. 2, p. 354- 360, June 2013.

25. Santana CJM, Lopes GT. O cuidado especializado do egresso da residência em enfermagem do Instituto Nacional de Câncer - INCA. Esc. Anna Nery Rev Enferm, Rio de Janeiro, v.11, n.3, p.417-22, set. 2007.

26. Cofen, Conselho Federal de Enfermagem. Resolução n 290 de 2004. Fixa as especialidades de Enfermagem. Resoluções COFEN, Brasília, DF.

27. Cofen, Conselho Federal de Enfermagem. Resolução no 259 de 2001. Estabelece Padrões mínimos para registro de Enfermeiro Especialista, na modalidade de Residência em Enfermagem. Resoluções COFEN, Brasília, DF.

28. Who. World Health Organization. National cancer control programmes: policies and managerial guidelines. 2 edition. Geneva: WHO; 2002.

29. Conselho Nacional de Saúde. Resolução n 358, de 15 de outubro de 2009. Diário Oficial da União, Brasília, DF, 23 out. 2009.

30. Machado KDG, Pessini L, Hossne WS. A formação em cuidados paliativos da equipe que atua em unidade de terapia intensiva: um olhar da bioética. Centro Universitário São Camilo, 1(1):34-42, 2007.

31. Mendonça ACA, Moreira MC, Carvolho V. Atenção paliativa oncológica em Unidade 
de Terapia Intensiva: um estudo da produção científica da enfermagem. Esc. Anna Nery, Rio de Janeiro, v. 16, n. 4, Dec. 2012.

32. North American Nursing Diagnosis Association - Diagnósticos de Enfermagem da NANDA: Definições e Classificação 2012-2014 - Editora Artmed.

33. Steensma DP, Loprinzi CL. The art and science of prognosis in patients with advanced cancer. European Journal of Cancer 2000 Oct;36(16):2025-7.

34. Sales CA, Silva MRB, Borgognoni K, Rorato C, Oliveira WT. Cuidado paliativo: a arte de estar-com-o-outro de uma forma autêntica. Revista de Enfermagem da UERJ, Rio de Janeiro, v. 16, n. 2, p. 174-9, abr./jun. 2008.

35. Cofen, Conselho Federal de Enfermagem. Resolução nº 261 de 2001. Fixa normas para registro de Enfermeiro, com pós-graduação. Resoluções COFEN, Brasília, DF.

36. Souza AS, Valadares GV. Desvelando o saber/ fazer sobre diagnósticos de enfermagem: experiência vivida em neurocirurgia oncológica. Rev. bras. enferm., Brasília, v. 64 , n. 5 , p. $890-897$, Oct. 2011.

37. Setz VG, D'innocenzo M. Avaliação da qualidade dos registros de enfermagem no prontuário por meio da auditoria. Acta paul. enferm., São Paulo , v. 22, n. 3, p. 313-317, 2009.

38. Galdeano LE et al. Diagnóstico de enfermagem de pacientes no período transoperatório de cirurgia cardíaca. Rev. Latino-Am. Enfermagem, Ribeirão Preto, v. 11, n. 2, p. 199-206, 2003.

39. Silva KS, Kruse MHL. Em defesa da sociedade: a invenção dos cuidados paliativos e a produção de subjetividades. Rev. esc. enferm. USP, São Paulo, v. 46, n.2, Apr. 2012.

40. Glare P, Sinclair CT. Palliative Medicine Review: Prognostication. Journal of Palliative Medicine 2008 Jan;11(1):84-103.

41. Scarpi E, Maltoni M, Miceli R, et al. Survival prediction for terminally ill cancer 
patients: revision of the palliative prognostic score with incorporation of delirium. Oncologist.2011;16:1793-1799.

42. Glare P, Virik K, Jones M, Hudson M, Eychmuller S, Simes J, et al. A systematic review of physicians' survival predictions in terminally ill cancer patients. BMJ 2003 Jul 24;327(7408):195.

43. Linhares AA. O raciocínio clínico do enfermeiro na avaliação de feridas em clientes com afecções oncológicas (Dissertação de Mestrado), 2010.

44. Sousa RM et al. Diagnósticos de enfermagem identificados em pacientes oncohematólogicos: mapeamento cruzado. Esc. Anna Nery, Rio de Janeiro, v. 19, n. 1, p. 54-65, Mar. 2015.

45. Pirovano M, Maltoni M, Nanni O, et al. A new palliative prognostic score: a first step for the staging of terminally ill cancer patients. Italian Multicenter and Study Group on Palliative Care. J Pain Symptom Manage. 1999;17:231-239. 